\title{
Investigation of Electron Beam Deposition Parameters Within a Scanning Electron Microscope
}

\author{
William Kellogg ${ }^{1}$, Benjamin D. Myers ${ }^{1,2}$, Karl Hujsak $^{1}$ and Vinayak P. Dravid ${ }^{1,2}$ \\ 1. Department of Materials Science \& Engineering, Northwestern University, Evanston, IL. \\ 2. NUANCE Center, Northwestern University, Evanston, IL.
}

The proliferation of aberration-corrected microscopes has facilitated imaging and spectroscopy of structures at the atomic scale. This high-resolution information has both provided new capabilities for investigating spatial and structural information and highlighted the issues resulting from beam-specimen interactions. The high energy electrons and high current densities required to resolve such fine structures result in ionizing radiation that produces atomic rearrangements and chemical reactions within the sample commonly referred to as beam damage. Conventionally, one seeks to avoid beam damage as it changes the nature of the sample. However, recent studies have sought to utilize this radiative damage by probing the phenomena related to beam-specimen interactions such as crystallization or amorphization [1-4].

The advent of fluidic-cell electron microscopy extends these studies of beam-sample phenomena to liquid and gaseous environments not possible in traditional high-vacuum electron microscopy. By combining improvements in microfabricated fluidic cells with the precise dose control of electron beam lithography, one can elucidate the highly complex and less well-known details of beam-specimen interactions in liquid or gaseous environments. Recent work involving liquid phase focused electron beam induced deposition (LP-FEBID) has demonstrated successful nanolithographic patterning of palladium [1,2], copper [3] and platinum [4] in a scanning transmission electron microscope (STEM). Although the literature has shown patterning in a variety of materials, the precise mechanism of LP-FEBID is still a topic of debate [3].

The small (femtoliter) volumes and confined geometries required to achieve imaging for fluidic-cell studies in S/TEM restrict the possible concentration ranges and impact uniformity of reaction conditions in these experiments. Little work currently exists investigating the role these concentration effects could play in the FEBID process. Additionally, the precise impact that temperature may have on the deposition process is also challenging to examine due to difficulties achieving uniform temperature control in the confined volumes of the S/TEM fluidic cell. We have employed LP-FEBID within a scanning electron microscope utilizing a Pelletier stage with precise temperature control to inspect how these environmental variables may impact the deposition process. These variables are being systematically explored in tandem with other relevant properties such as electron dose level, electron dose rate, and membrane surface effects to wholly characterize the deposition parameter space.

These measurements also extend to different solution systems and analysis for alloy formation versus phase separation. The presentation will cover the prospects for development of phase equilibria or pseudophase diagram concepts for solution-based localized synthesis, to map against diverse process conditions. The controlled deposition of various liquid phase precursors can establish nanostructured architectures of metal alloy systems with a high degree of size and morphological control, thus allowing for nanoscale device fabrication, and revealing information about beam-specimen interactions within fluid [5]. 
References:

[1] R. R. Unocic et al, Nanoscale 8 (2016), p. 15581.

[2] P. Abellan et al, Langmuir 36 (2016), p. 1468.

[3] E. U. Donev et al, Nanoscale 3 (2011), p. 2709.

[4] S. Esfandiarpour, L. Boehme, J. T. Hastings, Nanotechnology 28, (2017), 125301.

[5] This work was sponsored by the Air Force Research laboratory under agreement number FA865015-2-5518 and the Air Force Office of Scientific Research under Award FA9550-12-1-0280. This work made use of the EPIC facility of Northwestern University's NUANCE Center, which has received support from the Soft and Hybrid Nanotechnology Experimental (SHyNE) Resource (NSF ECCS1542205); the MRSEC program (NSF DMR-1121262) at the Materials Research Center; the International Institute for Nanotechnology (IIN); the Keck Foundation; and the State of Illinois, through the IIN.
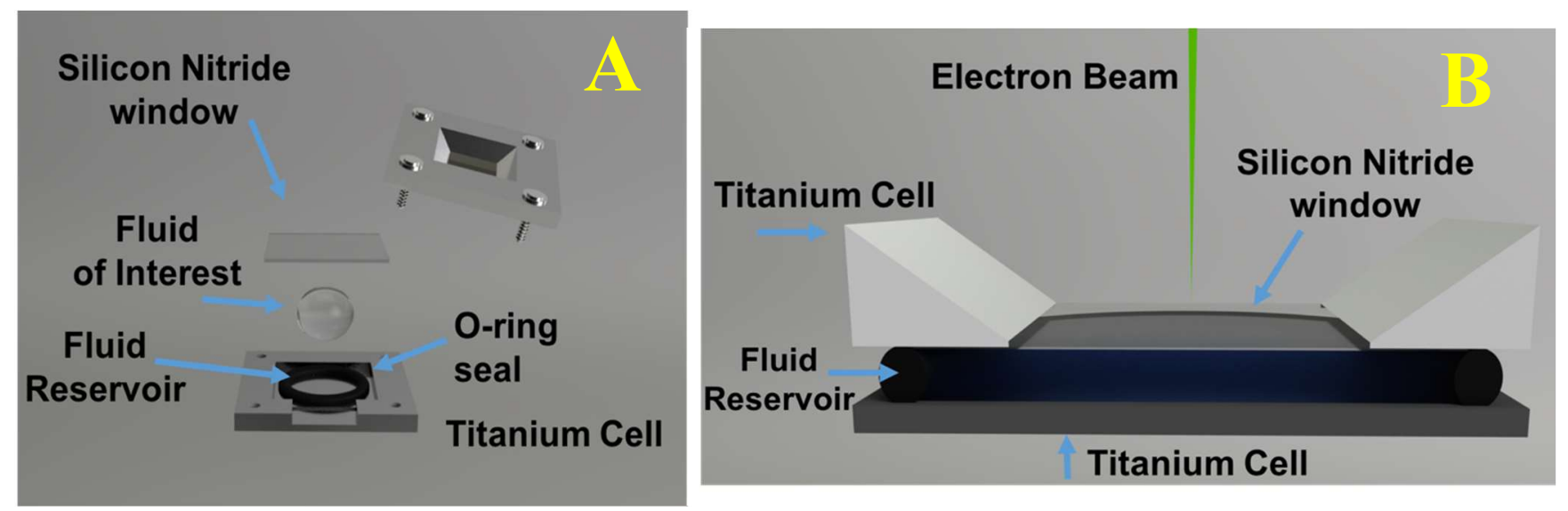

Figure 1. Top (A) and side (B) views of fluidic cell schematic for scanning electron microscope. Fluid is deposited via pipette in the fluidic reservoir. The incident electron beam is depicted as the green arrow; electrons are transmitted through the electron transparent silicon-nitride window, and some secondary and backscatter electrons exist for imaging.

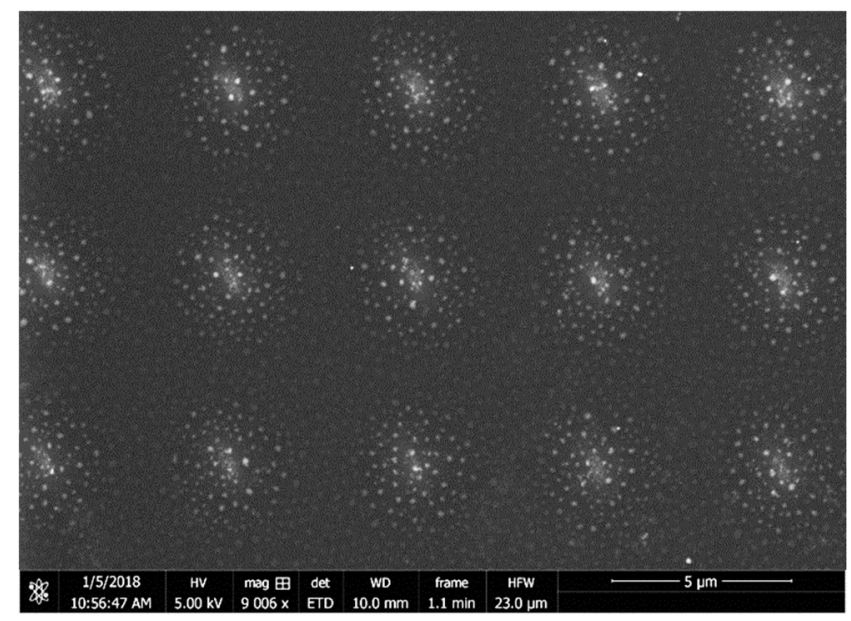

Figure 2. Palladium chloride deposition via liquid phase focused electron beam induced beam deposition, patterned and imaged in a FEI Quanta 650 FEG ESEM equipped with an NPGS lithography system. 